\title{
The state of multiple sclerosis: current insight into the patient/health care provider relationship, treatment challenges, and satisfaction
}

\author{
Mar Tintorél \\ Maggie Alexander ${ }^{2}$ \\ Kathleen Costello ${ }^{3}$ \\ Martin Duddy ${ }^{4}$ \\ David E Jones ${ }^{5}$ \\ Nancy Law 6 \\ Gilmore O'Neill ${ }^{7}$ \\ Antonio Uccelli ${ }^{8}$ \\ Robert Weissert ${ }^{9}$ \\ Sibyl Wray ${ }^{10}$
}

'Multiple Sclerosis Centre of Catalonia, Hospital Vall d'Hebron, Barcelona, Spain; ${ }^{2}$ European Multiple Sclerosis Platform, Brussels, Belgium; ${ }^{3}$ National Multiple Sclerosis Society, Denver, CO, USA; ${ }^{4}$ Royal Victoria Infirmary, Newcastle-upon-Tyne, UK; ${ }^{5}$ Department of Neurology, University of Virginia, Charlottesville, VA, USA; ${ }^{6}$ Nancy Law Consulting LLC, Parker, CO, USA; ' Biogen, Cambridge, MA, USA $;{ }^{8}$ Centre of Excellence for Biomedical Research, University of Genoa, Genoa, Italy; ' Department of Neurology, University of Regensburg, Regensburg, Germany; ${ }^{10} \mathrm{Hope}$ Neurology Multiple Sclerosis Center, Knoxville, TN, USA

This article was published in the following Dove Press journal:

Patient Preference and Adherence

22 December 2016

Number of times this article has been viewed

Background: Managing multiple sclerosis (MS) treatment presents challenges for both patients and health care professionals. Effective communication between patients with MS and their neurologist is important for improving clinical outcomes and quality of life.

Methods: A closed-ended online market research survey was used to assess the current state of MS care from the perspective of both patients with MS ( $\geq 18$ years of age) and neurologists who treat MS from Europe and the US and to gain insight into perceptions of treatment expectations/goals, treatment decisions, treatment challenges, communication, and satisfaction with care, based on current clinical practice.

Results: A total of 900 neurologists and 982 patients completed the survey, of whom $46 \%$ selfidentified as having remitting-relapsing MS, $29 \%$ secondary progressive MS, and $11 \%$ primary progressive MS. Overall, patients felt satisfied with their disease-modifying therapy (DMT); satisfaction related to comfort in speaking with their neurologist and participation in their DMT decision-making process. Patients who self-identified as having relapsing-remitting MS were more likely to be very satisfied with their treatment. Top challenges identified by patients in managing their DMT were cost, side effects/tolerability of treatment, and uncertainty if treatment was working. Half of the patients reported skipping doses, but only $68 \%$ told their health care provider that they did so.

Conclusion: Several important differences in perception were identified between patients and neurologists concerning treatment selection, satisfaction, expectations, goals, and comfort discussing symptoms, as well as treatment challenges and skipped doses. The study results emphasize that patient/neurologist communication and patient input into the treatment decisionmaking process likely influence patient satisfaction with treatment.

Keywords: health care provider survey, multiple sclerosis, patient-health care provider relationship, patient survey, treatment expectations, treatment satisfaction

\section{Introduction}

Multiple sclerosis (MS) is usually diagnosed in the third and fourth decades of life and can lead to fixed and/or progressive neurological impairment with a significant impact on activity. ${ }^{1,2}$ The last two decades have seen the advent of both disease-modifying therapies (DMTs) and new symptomatic therapies, adding to the complexity of the decision-making process of DMT selection and MS symptom management. ${ }^{3,4}$ More emphasis is being placed on patient-reported outcomes to help measure clinical outcomes, capture the full impact of the disease, and to determine health care provider (HCP) reimbursement. ${ }^{5,6}$ Thus, effective communication between patients and their 
HCPs becomes even more important for understanding the full impact of the disease to achieve optimal clinical outcomes.

Shared treatment decision making is promoted as representing good practice, and likely encourages treatment adherence and improves patient satisfaction. To be active participants in the DMT decision-making process, patients need to have adequate understanding of both their disease and the benefits/risks of treatments. ${ }^{7-9}$ Patients with MS who are well informed about their disease have significantly higher medication adherence than those who are not. ${ }^{10}$ The key element to maintaining treatment adherence in patients with MS is an open and honest relationship between the patient and HCP. ${ }^{11}$ Determining perceived differences in communication effectiveness between patients and their neurologists is essential for improving clinical outcomes and quality of life. ${ }^{12,13}$

The aim of this study was to assess the current state of MS care from the perspective of both patients with MS and neurologists treating patients with MS, and to gain insight into perceptions of treatment expectations/goals, treatment decisions, treatment challenges, communication, and satisfaction with care, based on current clinical practice.

\section{Methods Survey design}

Closed-ended questions for an online survey were developed to assess patient and neurologist attitudes, perceptions, and knowledge related to different aspects of MS, including the impact of disease on patients' lives, patient and HCP relationships, availability and usefulness of MS informational resources, and the expectations for satisfaction and also challenges encountered with a chosen DMT for MS. The survey was designed from a market research perspective for hypothesis-generating purposes. Questions were developed by the State of MS Consortium, comprising representatives from Biogen, GCI Health, and a steering committee (authors of this manuscript) of international MS experts and patient advocacy representatives, in collaboration with Harris Poll. Parallel questions for patients and neurologists were designed to allow comparisons. The survey responses were single or multiple response, numeric text, or rated on a 4- to 5-point Likert scale (eg, "very important", "important", "somewhat important", or "not at all important"; "strongly agree", "somewhat agree", "somewhat disagree", or "strongly disagree", respectively). As the nature of this online survey was blinded and noninterventional, formal review and approval by the ethics review committee of the Hospital Vall d'Hebron was not required for this study. The study was carried out in accordance with the principles outlined in the Declaration of Helsinki.

\section{Selection and recruitment}

Neurologists and patients from Italy, Germany, Spain, the UK, and the US were surveyed between March 18 and April 25, 2014.

\section{Patients}

Recruitment targets were set at 100-500 patients per country and recruitment continued until targets were met. Patients ( $\geq 18$ years of age) who participated in the research were recruited from survey panels provided by Harris Poll and vetted partners. Those invited to participate from the panels had previously agreed to participate in survey research, completed a double opt-in process, and identified themselves as having MS. Participating panel members were originally recruited into the survey panels using multiple methods (eg, directly, via banner ads, through other research). For this study, panelists were invited to participate in the research via email invitations. As access to the study was closed in each country once the recruitment target was met, determining the responder rate was not possible. A total of 2,023 panelists began the survey, with the 982 who completed it forming the analyzed cohort. Of those 1,047 invited panelists who started the survey but did not complete it, only 78 were determined to have met the age and diagnosis qualification criteria. The survey closed once the recruitment targets were met, so the responder rate could not be determined. Completion of the online survey questions deemed the qualify patients consented to participate in this study.

\section{Neurologists}

For neurologists treating patients with MS, recruitment targets were set at 150-200 neurologists per country and recruitment continued until the targets were met. US neurologists were randomly selected from the database of the American Medical Association master file. Those selected received a postal mail letter inviting them to participate in the research and were provided a link and password to the online survey. European neurologists were originally recruited to survey panels in a manner similar to patients from survey panels provided by Harris Poll and vetted partners and invited to participate in this survey via email. Neurologists had to have seen $\geq 1$ patient with MS, on average, each year in their practice to be included in the survey. A total of 1,124 participants began the survey, with 900 who completed it forming the analyzed cohort. Of those 224 invited participants who started the survey but did not complete it, 80 were determined to have met the inclusion criteria. The survey closed once the recruitment targets were met, so the responder rate could not be determined. Completion of the online survey questions deemed the qualify neurologists consented to participate in this study. 
For both neurologists and patients, specific subcategory quotas (eg, MS type, years in practice) within each country and audience were not predefined; thus, baseline demographics may have varied by country.

\section{Statistical analysis}

In the US, patient results were weighted for sex, age, region, race/ethnicity, education, and income to align with the US MS patient population based on predetermined targets. The targets were based on a demographic profile derived from the polling company. Neurologist results were weighted for sex, region, and years in practice. US neurologist results were weighted to be representative of the American Medical Association neurologist population. No other countries were weighted individually because information on the population profiles was not available. Additional weights were applied to the total data to give each country equal representation in the overall results.

Descriptive statistics are reported for the total patient/ neurologist samples. Given the aim of the project was to identify themes that might justify further study, no correction was made for multiple comparisons. Statistically significant differences across the subgroups of interest for patients or neurologists were determined by an independent sample $t$-test of column proportions and means at the $95 \%$ confidence level. No statistical testing was performed across audiences because the question phrasing differed between patients and neurologists. The dataset results were reviewed and any trends and patterns for themes of interest have been presented.

\section{Results}

\section{Demographics}

Nine hundred neurologists (Table S1) and 982 patients (Table 1) completed the survey.

\section{Patients}

Patients surveyed most commonly identified themselves as having relapsing-remitting MS (RRMS; 46\%), followed by secondary progressive MS (SPMS; 29\%) and primary progressive MS (11\%).

Baseline characteristics differed by country. More participating patients in the US self-identified as having RRMS than other countries, while more patients in Europe self-identified as having any form of progressive disease compared with the US (Table 1). Patients from the US and UK were older, on average, than patients from Germany, Italy, and Spain and subsequently reported a longer average time since MS diagnosis (Table 1). Overall, $62 \%$ of patients had at some stage taken a DMT for MS (63\% for RRMS, $68 \%$ for SPMS, and $52 \%$ for all others, including primary progressive MS, progressive relapsing MS [PRMS], and other/those who were not sure).

\section{Relationship and communication with HCPs}

The majority of patients somewhat/strongly agreed that their neurologists were honest in setting expectations for therapy success (85\%), had a good understanding of all aspects of MS (84\%), and had an open dialogue with them (82\%). Although most patients (83\%) reported that they were somewhat/very comfortable speaking with the neurologist or most frequently seen HCP about MS, nearly 1:5 patients reported being uncomfortable speaking about difficulty walking (19\%), tremors (19\%), and muscle spasms $(18 \%$; Table S2). Sensitive symptoms such as sexual difficulties (54\%), bladder/bowel problems (28\%), mood swings (26\%), difficulty swallowing (21\%), and cognitive/memory issues/trouble concentrating (21\%) emerged as the most

Table I Patient demographics

\begin{tabular}{|c|c|c|c|c|c|c|}
\hline Characteristics & Germany & Italy & Spain & UK & US & Total \\
\hline Patients (n) & 146 & 100 & 100 & 125 & 511 & 982 \\
\hline \multicolumn{7}{|l|}{ Type of MS (self-reported), \% } \\
\hline RRMS & 43 & 40 & 42 & 41 & $66^{\mathrm{a}-\mathrm{d}}$ & 46 \\
\hline SPMS & $26^{e}$ & $35^{\mathrm{e}}$ & $42^{\mathrm{a}, \mathrm{d}, \mathrm{e}}$ & $26^{\mathrm{e}}$ & 18 & 29 \\
\hline PPMS & 11 & 10 & 9 & $17^{\mathrm{e}}$ & 8 & 11 \\
\hline PRMS & $13^{b-e}$ & 5 & 4 & 6 & 4 & 6 \\
\hline Other/not sure & 7 & 10 & 3 & 10 & 5 & 7 \\
\hline Mean (SD) age (years) & $44(10)^{c}$ & $42(12)$ & $40(10)$ & $49(13)^{\mathrm{a}-\mathrm{c}}$ & $50(13)^{\mathrm{a}-\mathrm{c}}$ & $45(12)$ \\
\hline Female, $\%$ & $7 I^{c}$ & $59^{c}$ & 44 & $74^{\mathrm{b}, \mathrm{c}}$ & $69^{c}$ & 63 \\
\hline Mean (median) time since MS diagnosis (years) & II.8(I0) $)^{b, c}$ & $8.6(5)$ & $6.9(5)$ & $12.3(10)^{b, c}$ & $14.3(12)^{\mathrm{a}-\mathrm{c}}$ & $10.8(8)$ \\
\hline \multicolumn{7}{|l|}{ Participation in clinical trial for MS, \% } \\
\hline Yes, currently participating & $9^{d}$ & $13^{\mathrm{d}, \mathrm{e}}$ & $10^{\mathrm{d}, \mathrm{e}}$ & 2 & 5 & 8 \\
\hline Yes, participated in the past but not currently & 13 & $24^{\mathrm{a}, \mathrm{e}}$ & $28^{\mathrm{a}, \mathrm{e}}$ & $19^{e}$ & 10 & 19 \\
\hline No, I have never participated & $78^{\mathrm{b}, \mathrm{c}}$ & 63 & 62 & $79^{b, c}$ & $85^{\mathrm{b}, \mathrm{c}}$ & 74 \\
\hline
\end{tabular}

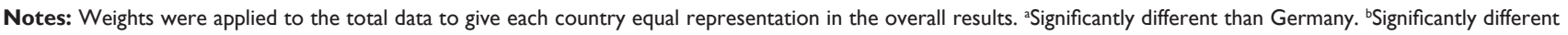
than Italy. 'Significantly different than Spain. `Significantly different than the UK. eSignificantly different than the US.

Abbreviations: MS, multiple sclerosis; PPMS, primary progressive multiple sclerosis; PRMS, progressive relapsing multiple sclerosis; RRMS, relapsing-remitting multiple sclerosis; SD, standard deviation; SPMS, secondary progressive multiple sclerosis. 
uncomfortable for patients to discuss. Only $2 \%-3 \%$ of neurologists identified symptoms such as difficulty in walking, tremors, and spasms as uncomfortable topics for their patients; however, they may have overestimated patients' discomfort talking about those same sensitive symptoms, including sexual difficulties (87\%), bladder/bowel problems (54\%), mood swings (37\%), and cognitive/memory issues/ trouble concentrating (37\%; Table S2).

Communication appeared to be important in treatment satisfaction, as those who were satisfied with their DMT were more likely than those who were less satisfied to say that they were very comfortable speaking to their neurologist (64\% vs $43 \%$, respectively; $P<0.05$ ) and were more likely to say they had an open dialogue with their HCP in which they could ask any questions they wanted (92\% vs $80 \%$, respectively; $P<0.05)$. The majority of patients somewhat/strongly agreed that their neurologist was accessible when needed (71\%).

\section{Cognitive impairment}

More neurologists (74\%) than patients (44\%) felt MS has had a negative impact on the patients' ability to perform cognitive tasks (Figure S1). When asked about MS symptoms they had experienced, 39\% of patients reported cognitive/ memory issues/trouble concentrating (Table S3). Thirtyseven percent of neurologists perceived their patients were not comfortable discussing cognitive impairment, whereas only $21 \%$ of patients were not comfortable discussing the problem with their neurologist (Table S2). While nearly all neurologists (95\%) felt maintaining cognitive function was a very important/important part of their patients' treatment goal, only $41 \%$ of patients who were currently taking DMTs reported it as part of their treatment goal.

\section{Treatment selection, satisfaction, and expectations}

Thirty-seven percent of patients reported that their HCP was the sole/primary DMT decision maker (Figure 1A), despite $71 \%$ of neurologists indicating that most patients with MS prefer that they, the neurologist, decide which treatment is appropriate for them. The level of patient participation in the treatment decision-making process remained constant across MS disease subtypes (Figure 1A). Patients in Spain $(n=55)$ were the most likely (42\%) to say their HCP was the sole decision maker compared with only $9 \%$ in the UK $(n=47)$, $10 \%$ in the US $(n=353), 14 \%$ in Italy $(n=63)$, and $15 \%$ in Germany ( $n=125$; Figure S2). Country-level results with small base size should be interpreted as directional in nature. Patients who were satisfied with their DMT were more likely to say that their expectations were very closely met than those who were not satisfied (67\% vs 44\%, respectively; $P<0.05$ ). More than half of patients who were currently taking DMTs (58\%) were very satisfied/satisfied and $42 \%$ were somewhat/not at all satisfied (Figure 1B), but this varied by country. US patients were the most likely to be satisfied with their MS medication(s) and patients in Italy less likely to be satisfied than patients in most other countries (Figure S3). Most patients (69\%) were optimistic about their future, and these patients were more likely to say they were very satisfied/satisfied with their current DMT (66\%) than those who were not optimistic (37\%). Patients who self-identified as having RRMS were more likely to be very satisfied with their current DMT than those who identified as having SPMS or other types of MS (31\% vs $17 \%$ and $16 \%$, respectively; $P<0.05)$. Patients who were satisfied with their DMT were more likely than others to report having been an equal partner or primary/sole decision maker in choosing their DMT (69\% vs 58\%, respectively; $P<0.05$ ). Most neurologists $(92 \%)$ agreed that MS patients who provided input when choosing a treatment plan tended to be more satisfied with their treatment experience than those who did not.

Only approximately half of patients (53\%) said that their DMT met the expectations set forth before treatment closely/very closely. More than 1:5 patients either said no expectations were set $(12 \%)$ or they could not recall if they were set $(10 \%)$.

\section{Treatment choice and goals}

Both patients and neurologists included safety and side effects as top factors that are important when making decisions about DMTs (Figure S4). Patients' top treatment goals for their current DMT included reducing relapse frequency (61\%), slowing disability progression (60\%), feeling better $(53 \%)$, and reducing relapse intensity (50\%). Similarly, neurologists' most important treatment goals for their patients' current MS DMT were reducing relapse frequency (97\% very important/important), slowing disability progression (97\%), feeling better (92\%), and reducing relapse intensity (93\%).

\section{Treatment challenges}

Top challenges identified by patients in managing their DMT were cost, side effects/tolerability of treatment, and uncertainty if treatment was working (Figure 2). Personal cost was identified as a challenge by more patients in the US than in Germany and the UK (36\% vs 23\% and 9\%, respectively). Patients and neurologists were aligned in identifying top challenges, with the exception of cost, but differed in the frequency of their response (Figure 2). However, approximately half of neurologists also cited lack of acceptance of the disease, while only a few patients themselves reported this. 


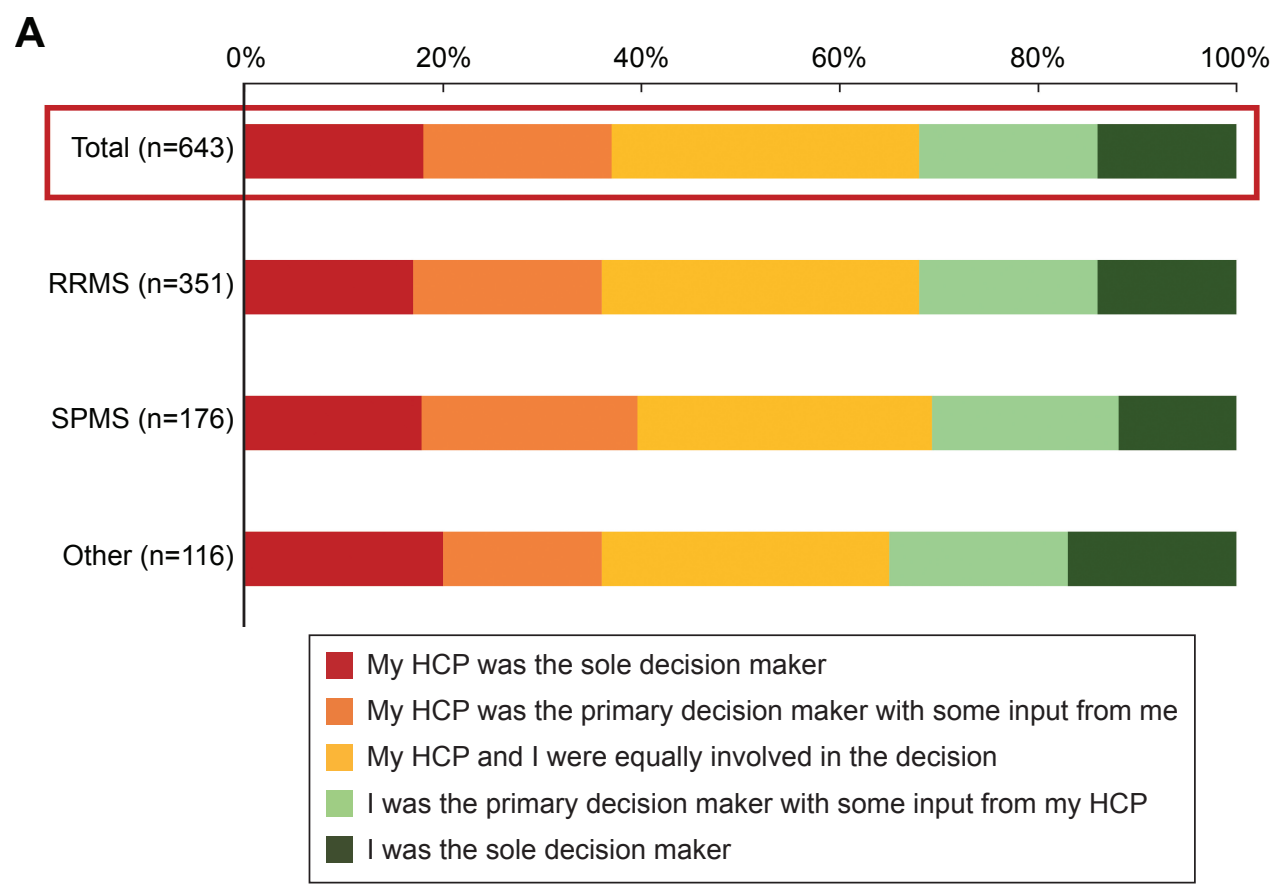

B

Very satisfied

Satisfied

Somewhat satisfied

Not at all satisfied
Total

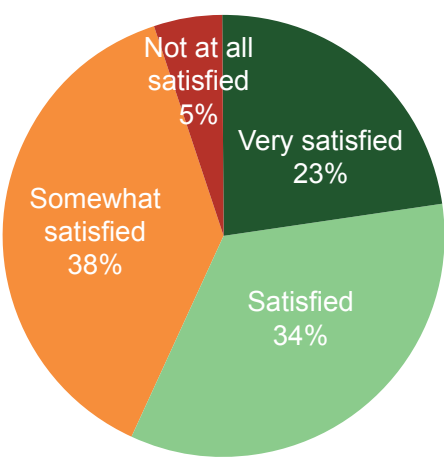

SPMS

( $n=114)$

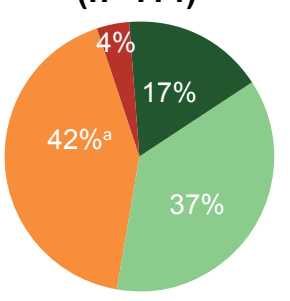

Other $(n=81)^{b}$

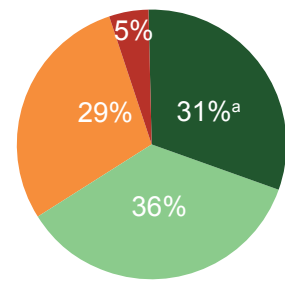

Figure I (A) Participation in DMT decision making and (B) satisfaction of patients with DMT.

Notes: (A) Qualified patients with MS who had taken prescription medications responded to the question, "How much input did you have in choosing your current/past disease-modifying medication(s), compared to your health care provider?" (B) $n=463$ patients with MS who were currently taking prescription medication responded to the question, "Overall, how satisfied are you with your current disease-modifying multiple sclerosis medication(s)?" The other category includes PPMS, progressive relapsing multiple sclerosis, and other/not sure. alndicates RRMS significantly different from SPMS and other, or SPMS and other significantly different from RRMS ( $<<0.05)$. ${ }^{\text {bPlease }}$ note small base size; results should be interpreted as directional in nature. Total may exceed $100 \%$ due to rounding.

Abbreviations: DMT, disease-modifying therapy; HCP, health care provider; MS, multiple sclerosis; PPMS, primary progressive multiple sclerosis; RRMS, relapsing-remitting multiple sclerosis; SPMS, secondary progressive multiple sclerosis.

\section{Skipped doses}

Nearly half of patients (49\%) reported never skipping doses of their DMT. Of the $51 \%$ of patients who reported skipping doses, only $68 \%$ told their HCP when they skipped a dose.
The top reasons patients cited for skipping a DMT dose were forgot (43\%), side effects $(22 \%)$, and feeling better $(15 \%$; Table 2). However, neurologists believed that side effects (73\%) were the main reason for skipping doses. 


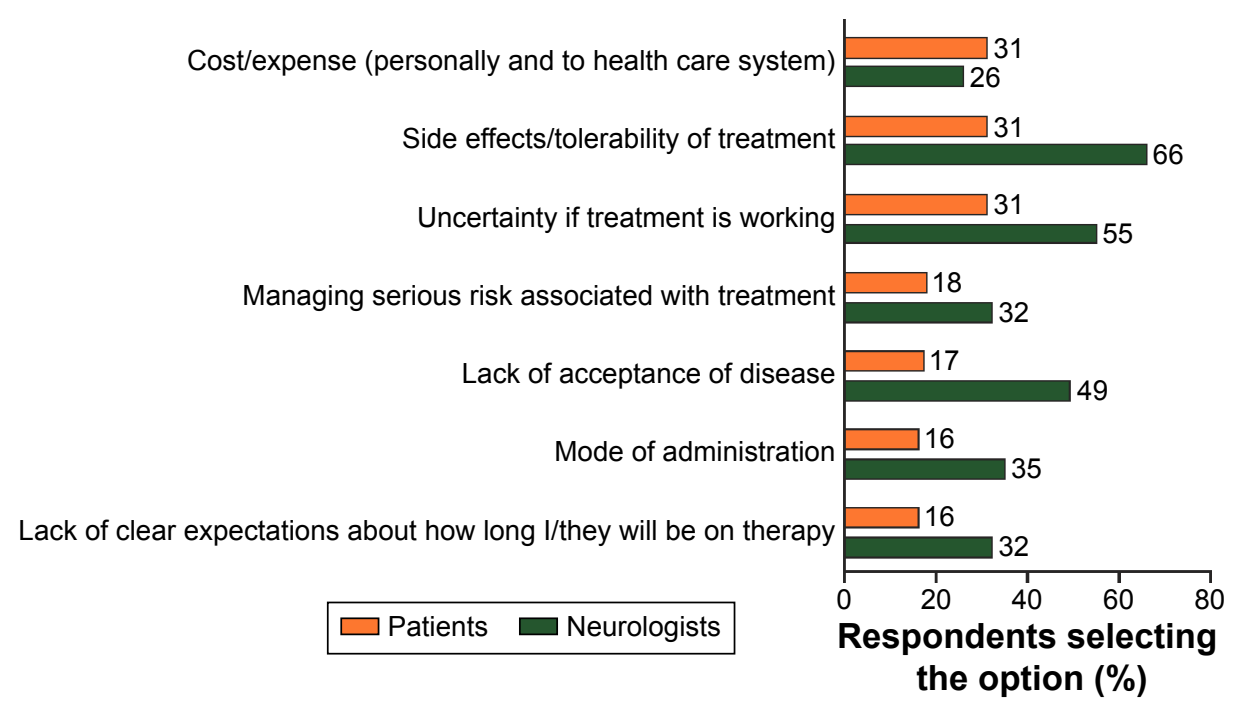

Figure 2 Top challenges for managing disease-modifying therapies for MS.

Notes: $\mathrm{n}=643$ qualified patients with MS who had taken prescription medicines responded to the question, "What are the greatest challenges you face in managing your disease-modifying multiple sclerosis treatment?" $n=900$ neurologists responded to the question, "What do you feel are the greatest challenges your patients face in managing their disease-modifying multiple sclerosis treatment?" The 7 of 15 most frequently selected items by both patients and neurologists are shown. The percentage of respondents selecting each option is indicated as a data label for each bar.

Abbreviation: MS, multiple sclerosis.

\section{Discussion}

This large global online survey evaluated MS patient and neurologist attitudes about treatment expectations and goals, treatment decisions, treatment challenges, communication, and satisfaction with care. Overall, patients and neurologists identified similar treatment goals and challenges. Patients who were satisfied with their current DMT were especially optimistic about the future. Patient input into their DMT decision-making process influences satisfaction, as does open communication, according to both patients and neurologists.

These results are consistent with another study that showed $79 \%$ of patients with MS preferred to take an active role in medical decision making. ${ }^{7}$ A shared medical

Table 2 Reasons for skipping prescribed dose(s) of medication

\begin{tabular}{ll}
\hline Reason (\%) & Total \\
& n=32 I \\
\hline I simply forgot & 43 \\
I was experiencing side effects & 22 \\
I was feeling better & I5 \\
Safety concerns & I3 \\
I did not think the treatment was working & I2 \\
Treatment did not make me feel better & II \\
I was no longer feeling better & I0 \\
I could no longer afford treatment & 8 \\
Reasons that were beyond my control & II \\
Other & II \\
Not sure/do not recall & 8 \\
\hline
\end{tabular}

Notes: All patients who had taken a disease-modifying therapy prescription medication and skipped doses answered the following question: "Which of the following, if any, are reasons why you skip doses of your disease-modifying medication? Please select all that apply." decision-making process regarding DMT choice is important in MS because current therapies are disease modifying, not curative, and adherence is important to optimize clinical outcomes. ${ }^{14}$ Studies have suggested that shortcomings in communication and information provided by HCPs may affect the quality of care, which in turn may affect treatment adherence, clinical outcomes, and ultimately quality of life. ${ }^{2,15}$ While high levels of satisfaction were noted in many aspects of MS management, important gaps were identified.

Key differences exist in the perceptions of patients and neurologists when discussing MS symptoms. Neurologists tend to overestimate their patients' discomfort talking about difficult topics such as sexual difficulties, bladder/bowel problems, and cognitive impairment, but tend to underestimate their discomfort discussing topics such as tremor and walking difficulty. These discrepancies could potentially lead to neurologists failing to identify and address modifiable symptoms. Cognition has been shown to be key in patients' quality of life and therefore it is a very relevant symptom. Cognitive impairment was of lower importance to patients than relapses and disability progression in most responses. The low number of patients identifying cognition as a problem may be related, at least in part, to the lack of correlation between perceived and objective cognitive issues, a disparity which has been previously noted. ${ }^{16-19}$ However, because cognition was not assessed in this survey, we cannot rule out that a higher proportion of patients without cognitive problems completed the survey. 
Clear communication between patients and HCPs is key for optimal MS care. ${ }^{12,13}$ For patients, understanding what treatment will, and perhaps more importantly, will not do is important. Nearly one-third of patients who skipped doses did not tell their HCP, reinforcing the fact that patient adherence should be routinely evaluated. Forgetting was cited at a similar rate in the Global Adherence Project observational study $(50 \%)^{20}$ as in this survey $(43 \%)$. Neurologists felt the main reason for non-adherence was side effects, indicating they are not aligned with patients on the main reasons for non-adherence. Identified treatment challenges also may affect adherence. For neurologists to effectively address non-adherence, they must understand patients' reasons for non-adherence, dose skipping patterns, and treatment challenges for individual patients.

\section{Limitations}

The results of this study should be interpreted with caution, particularly concerning the representativeness of the unweighted populations (ie, European patients and neurologists). The European results are only representative of the patients and neurologists who completed the survey and cannot reliably be extrapolated to the entire MS population/ treating neurologists. Although the translations were verified by native speakers, the multinational and multilingual nature of the survey may influence the results. Given the survey recruitment design and selection, response biases are possible and should be considered when interpreting the results. Patient recruitment targets were set for the total population per country without subtargets based on MS disease type; therefore, disease subtypes were not actively balanced across countries. This lack of balancing may be the reason for the unexpectedly high number of patients who identified as having PRMS in Germany compared with other countries or it may be an inconsistency in translation of the definition of PRMS. Also, we do not know which, if any, participating patients came from the same practices as the neurologists due to the independent recruitment of patients and neurologists. Therefore, the analysis represents both different perceptions and potentially different disease courses, treatment experiences, and patient-HCP interactions. Further, although the survey's online approach allowed for a relatively large (900 neurologists and $>900$ patients) sample and an opportunity for respondents to answer sensitive questions honestly without introducing the bias of an interviewer, a bias associated with respondent self-selection may exist. We do not have data on the percentage of patients and neurologists who completed the survey compared with those who received the survey. The survey also is limited in that cognition was not assessed so, potentially, only patients with less cognitive impairment completed the survey. Finally, the differences in the importance and role of treatment cost between countries should be interpreted with caution, because the cause of these differences is likely due to the different health care systems. Along with costs, different health care systems also can bias perceptions with regard to treatment satisfaction and value of care. ${ }^{21}$

\section{Conclusion and recommendations}

The results presented offer insight into differences between the perceptions of patients and neurologists and highlight differences in priorities for choosing a MS therapy. The study was aimed at identifying themes that might warrant further study with more robust methodology and was not in itself aiming to provide definitive statistics. Providing information that raises awareness of the importance of patient/neurologist collaboration and actions that can enhance these relationships should be considered. Therefore, a collaborative approach for setting appropriate treatment expectations and greater patient input in therapy choice is key for improving treatment satisfaction and adherence.

Based on these data, some action points were identified: 1) for HCPs, develop effective tools to improve communication, improving recognition of patients' priorities, including patient-related outcomes; 2) for patients, develop educational programming that aids in learning about the disease and how to more actively participate in all aspects of decision making, thus working to become better self-advocates; and 3) opportunities for professional organizations (eg, European Academy of Neurology, American Academy of Neurology) and patient organizations (eg, European Multiple Sclerosis Platform) to work together to raise awareness of discrepancies in perceptions that exist between patients and HCPs.

\section{Acknowledgments}

Biogen provided funding for medical writing support in the development of this paper; Karen M Spach wrote the first draft of the manuscript based on input from authors, and Kristen DeYoung copyedited and styled the manuscript per journal requirements. Biogen reviewed and provided feedback on the paper to the authors. The authors had full editorial control of the paper, and provided their final approval of all content. Harris Poll (Robyn Bell Dickson and colleagues) assisted with survey design and support, data collection, and data analysis. Statistical analysis was conducted by Harris Poll. 
This manuscript has not been previously published and is not under consideration elsewhere. Some of these data were presented at the Joint Americas Committee for Treatment and Research in Multiple Sclerosis (ACTRIMS) European Committee for Treatment and Research in Multiple Sclerosis (ECTRIMS) Meeting, September 10-13, 2014, Boston, MA, USA, the 67th American Academy of Neurology (AAN) Annual Meeting, April 18-25, 2015, Washington, DC, USA, and the 31st Congress of the ECTRIMS Meeting, October 7-10, 2015, Barcelona, Spain. The abstracts were published on the ECTRIMS website and in the AAN supplement in Neurology (Wray S, Alexander M, Duddy M, Jones DE, Law N, O’Neill G, Uccelli A, Weissert R, Tintoré M. The state of multiple sclerosis survey: relationship of expectations, decision-making, communication, and satisfaction with treatment. Neurology. 2015;84[14 Suppl]:P3.235).

\section{Author contributions}

Mar Tintoré, Maggie Alexander, Martin Duddy, David E Jones, Nancy Law, Gilmore O’Neill, Antonio Uccelli, Robert Weissert, and Sibyl Wray participated in the State of MS Consortium, which designed the survey questions. Mar Tintoré, Maggie Alexander, Kathleen Costello, Martin Duddy, David E Jones, Nancy Law, Gilmore O'Neill, Antonio Uccelli, Robert Weissert, and Sibyl Wray interpreted and critically assessed the data for inclusion in the manuscript. Mar Tintoré, Maggie Alexander, Kathleen Costello, Martin Duddy, David E Jones, Nancy Law, Gilmore O’Neill, Antonio Uccelli, Robert Weissert, and Sibyl Wray contributed to and critically revised the manuscript.

\section{Disclosure}

Mar Tintoré received consulting and non-CME service fees from Almirall, Biogen, EMD Merck Serono, Genzyme, Novartis, Roche, Sanofi-Aventis, and Teva UK Limited; educational/research support from Bayer HealthCare, Biogen, Genzyme, EMD Merck Serono, Novartis Pharma AG, and Teva UK Limited. Martin Duddy received honoraria, educational support, consulting fees, and research support from Bayer HealthCare, Biogen, Genzyme, Merck Serono, Novartis Pharma AG, Roche, and Teva UK Limited; and personal compensation as associate editor for the Multiple Sclerosis Journal. David E Jones received consulting fees from Biogen, Genzyme, and Novartis; and research support from Biogen. Nancy Law received consulting fees from Biogen in her current role; no consulting fees were received in her former role as an employee of the National Multiple Sclerosis Society (Denver, CO, USA). Gilmore O’Neill is a full-time employee of and holds stock/stock options in Biogen. Antonio Uccelli received consulting or speaker fees from Allergan, Bayer HealthCare, Biogen, Genzyme, Merck Serono, Novartis, Roche, and Teva; and research support from Biogen, Merck Serono, and Novartis. Robert Weissert received consulting fees from Biogen, Genzyme, Merck Serono, Novartis, Roche, and Teva; served on speakers bureaus for Ärztlicher Kreisverband Weiden and Biogen; and performed contracted research for Novartis. Sibyl Wray received consulting fees from Acorda, Biogen, EMD Serono, Genzyme, Novartis, Questcor, and Teva; served on speakers bureaus for Acorda, Bayer HealthCare, Biogen, EMD Serono, Genzyme, Novartis, Questcor, and Teva; and performed contracted research for Biogen, EMD Serono, Genzyme, Novartis, Receptos, and Roche. The authors report no other conflicts of interest in this work.

\section{References}

1. Culpepper WJ, Wallin MT, Magder LS, et al. VHA Multiple Sclerosis Surveillance Registry and its similarities to other contemporary multiple sclerosis cohorts. J Rehabil Res Dev. 2015;52(3):263-272.

2. National Institute for Health and Care Excellence. Multiple sclerosis in adults: management [published October 2014]. Available from: http://www.nice.org.uk/guidance/cg186. Accessed July 15, 2015.

3. Oh J, Calabresi PA. Emerging injectable therapies for multiple sclerosis. Lancet Neurol. 2013;12(11):1115-1126.

4. Ziemssen T, De Stefano N, Pia Sormani M, Van Wijmeersch B, Wiendl H, Kieseier BC. Optimizing therapy early in multiple sclerosis: an evidence-based view. Mult Scler Relat Disord. 2015;4(5):460-469.

5. Doward LC, Gnanasakthy A, Baker MG. Patient reported outcomes: looking beyond the label claim. Health Qual Life Outcomes. 2010;8:89.

6. Rae-Grant A, Bennett A, Sanders AE, Phipps M, Cheng E, Bever C. Quality improvement in neurology: multiple sclerosis quality measures: executive summary. Neurology. 2015;85(21):1904-1908.

7. Heesen C, Kasper J, Segal J, Köpke S, Mühlhauser I. Decisional role preferences, risk knowledge and information interests in patients with multiple sclerosis. Mult Scler. 2004;10(6):643-650.

8. Giordano A, Mattarozzi K, Pucci E, et al. Participation in medical decision-making: attitudes of Italians with multiple sclerosis. J Neurol Sci. 2008;275(1-2):86-91.

9. Solari A, Acquarone N, Pucci E, et al. Communicating the diagnosis of multiple sclerosis - a qualitative study. Mult Scler. 2007;13(6):763-769.

10. de Seze J, Borgel F, Brudon F. Patient perceptions of multiple sclerosis and its treatment. Patient Prefer Adherence. 2012;6:263-273.

11. Costello K, Kennedy P, Scanzillo J. Recognizing nonadherence in patients with multiple sclerosis and maintaining treatment adherence in the long term. Medscape J Med. 2008;10(9):225.

12. Lugaresi A, Ziemssen T, Oreja-Guevara C, Thomas D, Verdun E. Improving patient-physician dialog: commentary on the results of the MS Choices survey. Patient Prefer Adherence. 2012;6:143-152.

13. Koudriavtseva T, Onesti E, Pestalozza IF, Sperduti I, Jandolo B. The importance of physician-patient relationship for improvement of adherence to long-term therapy: data of survey in a cohort of multiple sclerosis patients with mild and moderate disability. Neurol Sci. 2012;33(3):575-584.

14. Lizán L, Comellas M, Paz S, Poveda JL, Meletiche DM, Polanco C. Treatment adherence and other patient-reported outcomes as cost determinants in multiple sclerosis: a review of the literature. Patient Prefer Adherence. 2014;8:1653-1664.

15. Thorne S, Con A, McGuinness L, McPherson G, Harris SR. Health care communication issues in multiple sclerosis: an interpretive description. Qual Health Res. 2004;14(1):5-22. 
16. Christodoulou C, Melville P, Scherl WF, et al. Perceived cognitive dysfunction and observed neuropsychological performance: longitudinal relation in persons with multiple sclerosis. J Int Neuropsychol Soc. 2005;11(5):614-619.

17. Maor Y, Olmer L, Mozes B. The relation between objective and subjective impairment in cognitive function among multiple sclerosis patients - the role of depression. Mult Scler. 2001;7(2):131-135.

18. Middleton LS, Denney DR, Lynch SG, Parmenter B. The relationship between perceived and objective cognitive functioning in multiple sclerosis. Arch Clin Neuropsychol. 2006;21(5):487-494.

19. Lovera J, Bagert B, Smoot KH, et al. Correlations of Perceived Deficits Questionnaire of Multiple Sclerosis Quality of Life Inventory with Beck Depression Inventory and neuropsychological tests. J Rehabil Res Dev. 2006;43(1):73-82.
20. Devonshire V, Lapierre Y, Macdonell R, et al; GAP Study Group. The Global Adherence Project (GAP): a multicenter observational study on adherence to disease-modifying therapies in patients with relapsingremitting multiple sclerosis. Eur J Neurol. 2011;18(1):69-77.

21. Bleich SN, Ozaltin E, Murray CK. How does satisfaction with the health-care system relate to patient experience? Bull World Health Organ. 2009;87(4):271-278. 


\section{Supplementary materials}

Table SI Neurologist demographics

\begin{tabular}{|c|c|c|c|c|c|c|}
\hline Characteristics & Germany & Italy & Spain & UK & US & Total \\
\hline Neurologists (n) & 200 & 200 & 150 & 150 & 200 & 900 \\
\hline \multicolumn{7}{|l|}{ Years in practice, $n(\%)$} \\
\hline$\leq 10$ & $80(40)^{\mathrm{b}, \mathrm{e}}$ & $57(29)$ & $50(33)^{\mathrm{e}}$ & $65(43)^{\mathrm{b}, \mathrm{e}}$ & $45(23)$ & $302(34)$ \\
\hline $1 \mathrm{I}-20$ & $78(39)^{b}$ & $59(30)$ & $67(45)^{\mathrm{b}, \mathrm{e}}$ & $6 I(4 I)^{b}$ & $63(32)$ & $334(37)$ \\
\hline $21-30$ & $36(18)$ & $59(30)^{\mathrm{a}, \mathrm{c}, \mathrm{d}}$ & $26(17)$ & $16(11)$ & $49(24)^{d}$ & $180(20)$ \\
\hline$\geq 31$ & $6(3)$ & $25(\mid 3)^{a, c, d}$ & $7(5)$ & $8(5)$ & $43(22)^{\mathrm{a}-\mathrm{d}}$ & $85(9)$ \\
\hline Mean (SD) age (years) & $47(8)^{c, d}$ & $47(10)^{\mathrm{c,d}}$ & $44(8)$ & $42(8)$ & $5 I(I I)^{a-d}$ & $46(10)$ \\
\hline Female, n (\%) & $39(20)$ & $76(38)^{\mathrm{a}, \mathrm{e}}$ & $52(35)^{\mathrm{a}}$ & $59(39)^{\mathrm{a}, \mathrm{e}}$ & $53(27)$ & $283(32)$ \\
\hline Average number of patients with MS seen in year & $194^{e}$ & $174^{\mathrm{e}}$ & $170^{e}$ & $235^{\mathrm{b}, \mathrm{c}, \mathrm{e}}$ & 124 & 180 \\
\hline
\end{tabular}

Notes: Percentages may not add to 100 due to rounding. Weights were applied to the total data to give each country equal representation in the overall results. aSignificantly different than Germany. 'bignificantly different than Italy. 'Significantly different than Spain. ¿Significantly different than the UK. eSignificantly different than the US.

Abbreviations: MS, multiple sclerosis; SD, standard deviation.

Table S2 Symptoms patients are not comfortable discussing with neurologist

\begin{tabular}{|c|c|c|}
\hline Symptoms (\%) & $\begin{array}{l}\text { Total patients } \\
\text { (variable) }\end{array}$ & $\begin{array}{l}\text { Total neurologists } \\
\mathrm{n}=900\end{array}$ \\
\hline Sexual difficulties & 54 & 87 \\
\hline Bladder/bowel problems & 28 & 54 \\
\hline Mood swings & 26 & 37 \\
\hline Difficulty swallowing & 21 & 4 \\
\hline Cognitive/memory issues/trouble concentrating & 21 & 37 \\
\hline Difficulty walking & 19 & 3 \\
\hline Tremors & 19 & 2 \\
\hline Muscle spasms & 18 & 2 \\
\hline Pain & 17 & 4 \\
\hline Vision problems & 17 & 3 \\
\hline Fatigue & 16 & 6 \\
\hline Dizziness/lightheadedness & 16 & 2 \\
\hline Speech problems & 16 & 5 \\
\hline Difficulties with fine motor tasks & 15 & 5 \\
\hline Impaired balance/tendency to fall & 15 & 4 \\
\hline Tingling/"pins and needles" sensations, itching & 14 & 2 \\
\hline Numbness/impaired sensation & 13 & 2 \\
\hline Impaired upper limb function & 12 & 2 \\
\hline None & $\mathrm{N} / \mathrm{A}$ & 8 \\
\hline
\end{tabular}

Notes: Survey question: "Which of the following symptoms, if any, are you not comfortable talking about with your neurologist you see the most for your multiple sclerosis?! Which of the following symptoms, if any, do you think your multiple sclerosis patients are not comfortable talking about with you? Please select all that apply." Each symptom is based on those who experienced symptom (and also saw a health care provider in the last year).

Abbreviation: N/A, not applicable. 


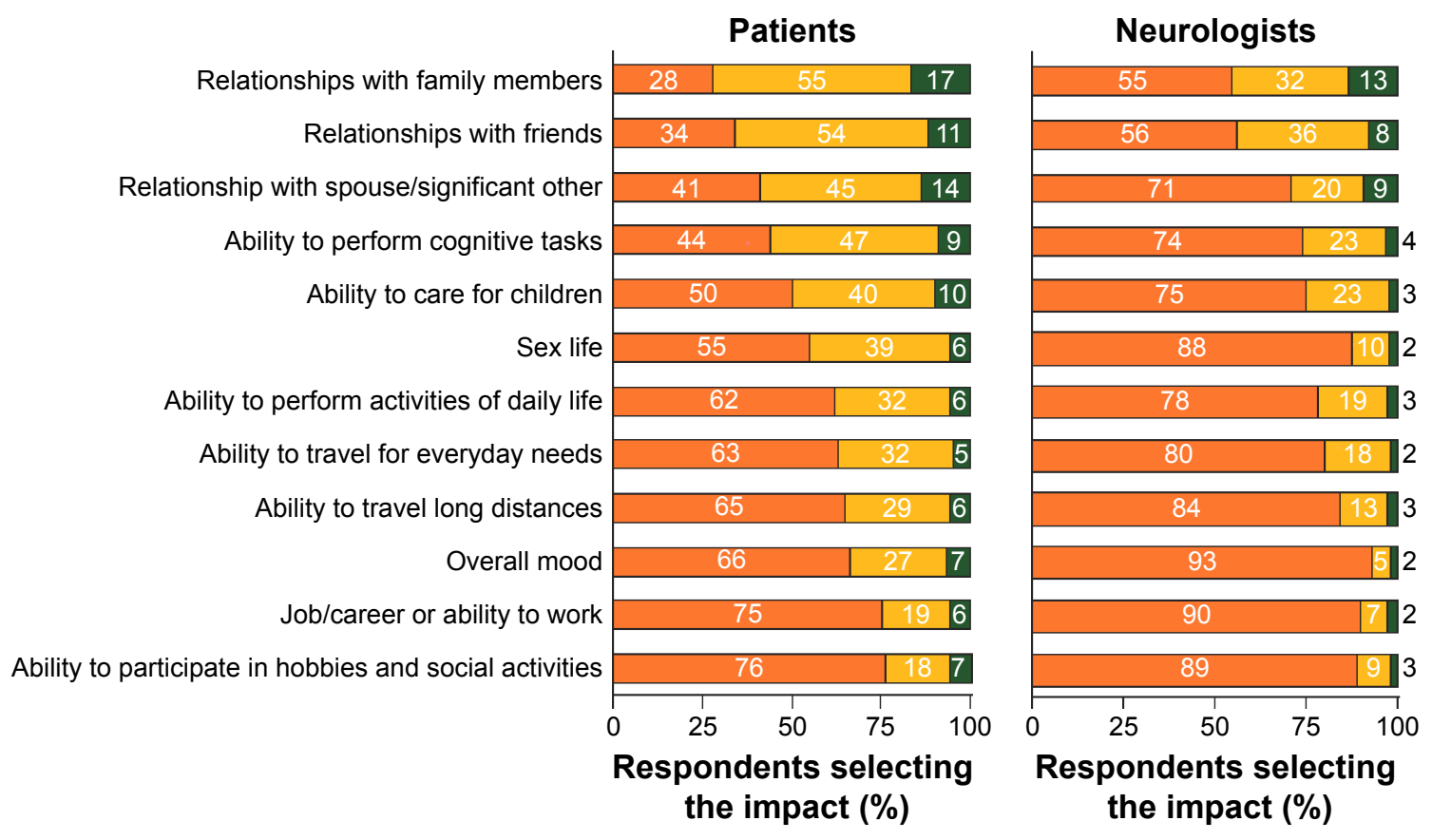

$\square$ Positive impact $\square$ No impact $\square$ Negative impact

Figure SI Impact of multiple sclerosis on the following aspects of your life/your patients' lives.

Notes: Survey question: "How much of an impact, if any, has having multiple sclerosis had on each of the following aspects of your life/your patients' lives?" Base: qualified patients who said the attribute was applicable; qualified neurologists who said they had an opinion on the attribute.

Table S3 Symptoms experienced

\begin{tabular}{|c|c|c|}
\hline Symptoms (\%) & $\begin{array}{l}\text { Have experienced }^{a} \\
n=982\end{array}$ & $\begin{array}{l}\text { Experience daily } \\
\text { (variable) }\end{array}$ \\
\hline Fatigue & 71 & 74 \\
\hline Tingling/“"pins and needles” sensations, itching & 59 & 54 \\
\hline Difficulty walking & 58 & 69 \\
\hline Numbness/impaired sensation & 56 & 56 \\
\hline Impaired balance/tendency to fall & 56 & 57 \\
\hline Pain & 50 & 59 \\
\hline Muscle spasms & 46 & 51 \\
\hline Mood swings & 45 & 46 \\
\hline Vision problems & 41 & 44 \\
\hline Impaired upper limb function & 40 & 46 \\
\hline Bladder/bowel problems & 40 & 70 \\
\hline Dizziness or lightheadedness & 39 & 38 \\
\hline Cognitive/memory issues/trouble concentrating & 39 & 61 \\
\hline Difficulties with fine motor tasks & 33 & 53 \\
\hline Sexual difficulties & 32 & 43 \\
\hline Tremors & 27 & 43 \\
\hline Speech problems & 20 & 43 \\
\hline Difficulty swallowing & 18 & 46 \\
\hline Other & 4 & 46 \\
\hline
\end{tabular}

Notes: aBase: all qualified patients who had experienced an applicable MS symptom. Survey question: "Which of the following symptoms of multiple sclerosis, if any, have you experienced? Please select all that apply." 'Base: all qualified patients who had experienced an applicable MS symptom. Survey question: "Thinking of the symptoms you have experienced, which of the following, if any, do you experience on a daily basis? Please think only of the symptoms you experience when you are not having a relapse. Please select all those that apply.

Abbreviation: MS, multiple sclerosis. 


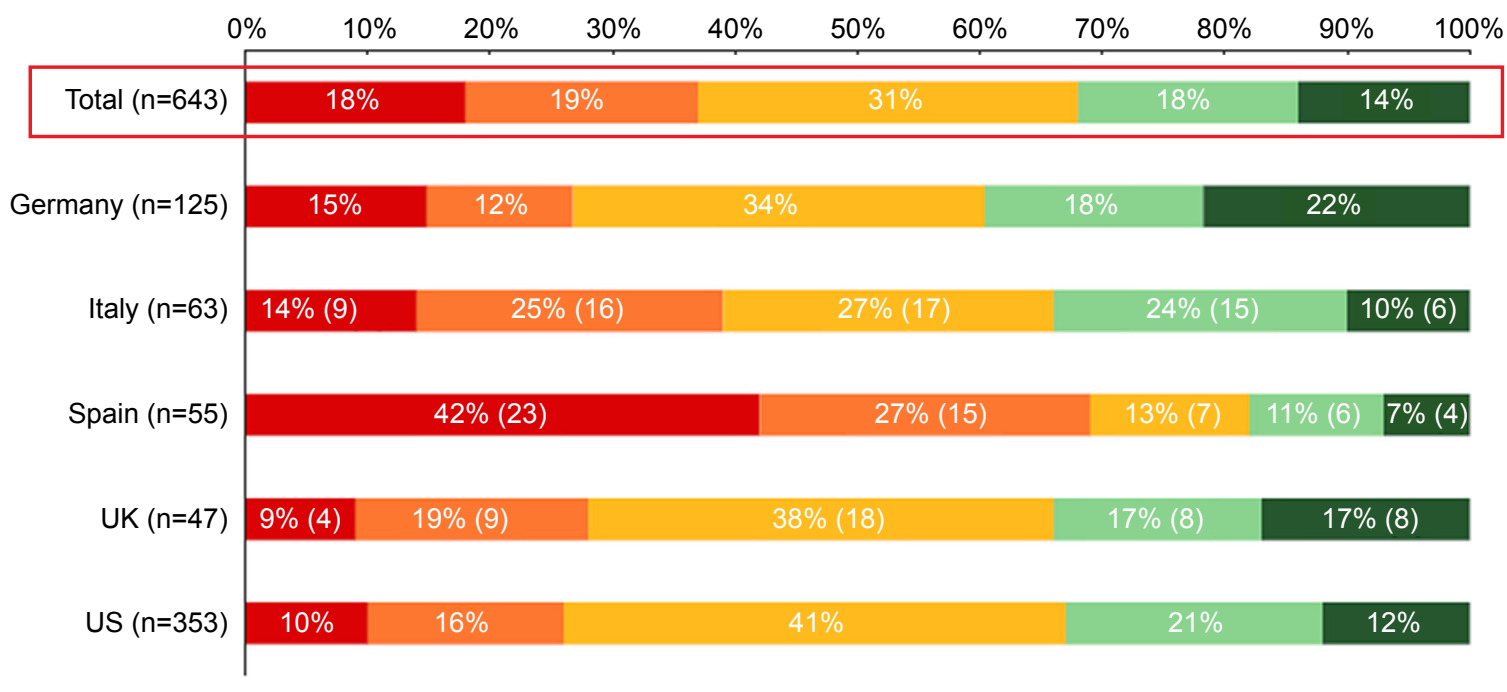

My HCP was the sole decision maker

My HCP was the primary decision maker with some input from me

My HCP and I were equally involved in the decision

$\square$ I was the primary decision maker with some input from my HCP

I was the sole decision maker

Figure S2 Patient participation in DMT decision making.

Notes: $\mathrm{n}=643$ qualified patients with MS who had taken prescription medications responded to the question, "How much input did you have in choosing your current/past disease-modifying medication(s), compared to your health care provider?" Percentages (number) are shown. Caution: country-level results with small base size should be interpreted as directional in nature and, as such, frequencies are shown in additon to the percentages where applicable.

Abbreviations: DMT, disease-modifying therapy; HCP, health care provider; MS, multiple sclerosis.
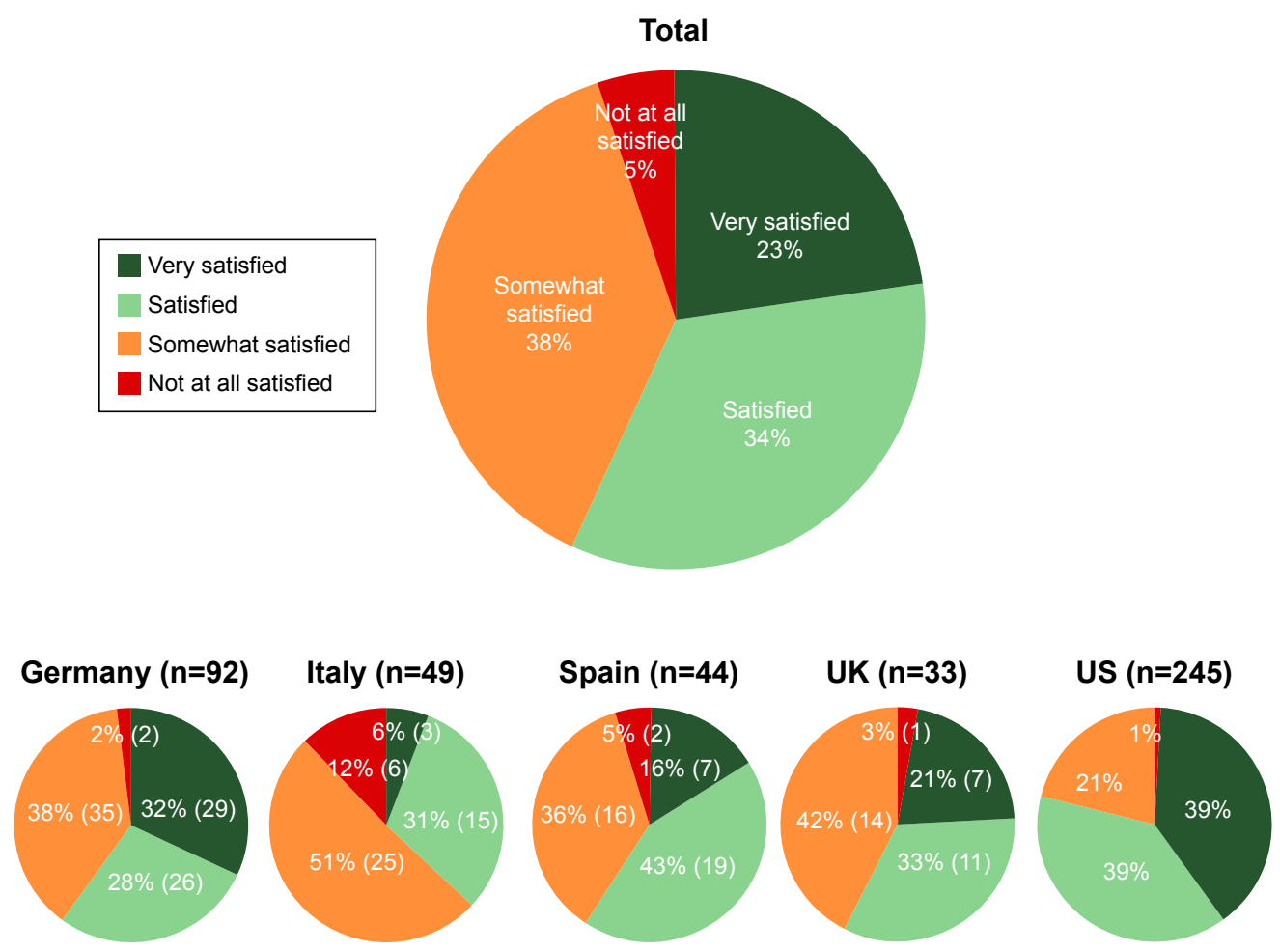

Figure S3 Patients' satisfaction with DMTs.

Notes: $n=463$ qualified patients with MS who were currently taking prescription medication responded to the question, "Overall, how satisfied are you with your current disease modifying MS medication(s)?" Percentages (numbers) are shown. Caution: country-level results with small base size should be interpreted as directional in nature and, as such, frequencies are shown in additon to the percentages where applicable.

Abbreviations: DMT, disease-modifying therapy; MS, multiple sclerosis. 


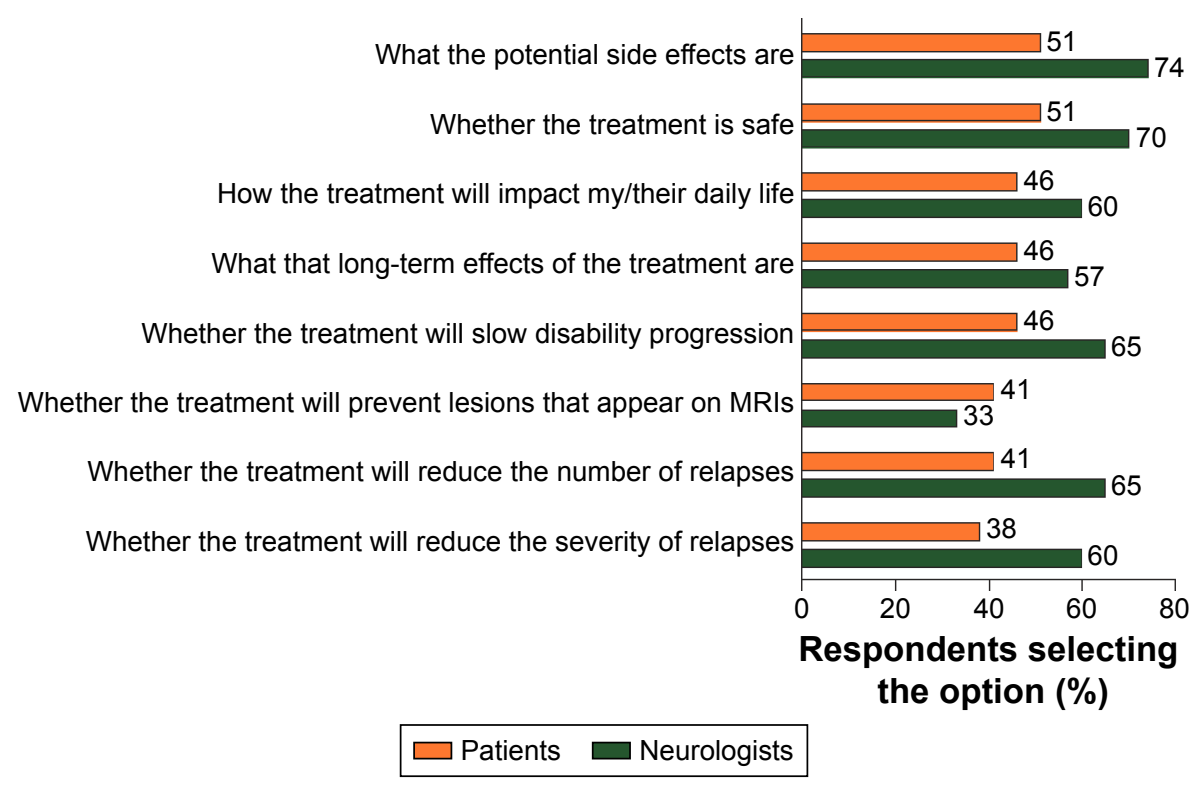

Figure S4 Top factors important for patients to know when making decisions about disease-modifying treatments for MS according to neurologists and patients. Notes: $\mathrm{n}=982$ qualified patients with MS responded to the question, "Which of the following, if any, are most important for you to know when making decisions about disease-modifying treatments?" $\mathrm{n}=900$ qualified neurologists responded to the question, "Which of the following, if any, are the most important for your MS patients to know when making decisions about their disease-modifying treatments?" The top 8 of 20 factors selected by patients are shown. The percentage of respondents selecting each option is indicated as a data label for each bar.

Abbreviations: MRI, magnetic resonance imaging; MS, multiple sclerosis.

Patient Preference and Adherence

\section{Publish your work in this journal}

Patient Preference and Adherence is an international, peer-reviewed, open access journal that focuses on the growing importance of patient preference and adherence throughout the therapeutic continuum. Patient satisfaction, acceptability, quality of life, compliance, persistence and their role in developing new therapeutic modalities and compounds to optimize

\section{Dovepress}

clinical outcomes for existing disease states are major areas of interest for the journal. This journal has been accepted for indexing on PubMed Central. The manuscript management system is completely online and includes a very quick and fair peer-review system, which is all easy to use. Visit http://www. dovepress.com/testimonials.php to read real quotes from published authors.

\footnotetext{
Submit your manuscript here: http://www.dovepress.com/patient-preference-and-adherence-journal
} 